\title{
Ocular Basidiobolomycosis: A Case Report
}

\author{
Napaporn Tananuvat ${ }^{a} \quad$ Sumet Supalaset ${ }^{a} \quad$ Muanploy Niparugs $^{a}$ \\ Siriporn Chongkae ${ }^{b} \quad$ Nongnuch Vanittanakom $^{b}$ \\ aDepartment of Ophthalmology, Faculty of Medicine, Chiang Mai University, \\ Chiang Mai, Thailand; 'bepartment of Microbiology, Faculty of Medicine, Chiang Mai \\ University, Chiang Mai, Thailand
}

\section{Keywords}

Basidiobolus ranarum · Infectious scleritis · Keratitis · Ocular basidiobolomycosis · Orbital cellulitis

\begin{abstract}
Background: Ocular basidiobolomycosis is an unusual infection caused by fungus of the order Entomophthorales. This fungus has been previously reported as a common cause of skin, subcutaneous, and gastrointestinal tract infection. The fungus isolation and its typical characteristics are clues for diagnosis of this uncommon pathogen. Case Report: A 47-year-old male patient with nodular scleritis in the left eye after an eye injury from sawdust was treated as bacterial scleritis. The lesion improved with early surgical drainage and antibacterial therapy; then, he was discharged from the hospital. Thereafter, the patient was re-admitted due to progression of infectious scleritis with keratitis and orbital cellulitis. Surgical abscess drainage was performed again. The microbiological study demonstrated Basidiobolus ranarum. The patient was treated with topical ketoconazole, subconjunctival fluconazole injection, and oral itraconazole with partial response to the treatment. However, the patient eventually denied any further treatment and did not return for follow-up. Conclusions: $B$. ranarum is a rare pathogen of ocular infection in which a definite diagnosis requires isolation of the causative organism. Delay in diagnosis and appropriate treatment can lead to extension of the infection and poor outcomes.




\section{Case Reports in Ophthalmology}

\section{Introduction}

Basidiobolomycosis is an unusual fungal infection caused by Basidiobolus species, member of the former Zygomycetes, subphylum Entomophthoromycotina, order Entomophthorales. It is known for skin and subcutaneous zygomycosis in healthy individuals [1,2]. This fungus can also infect other tissue including the gastrointestinal tract, lungs, maxillary sinus, palate, and turbinates [2]. In the past, pathogenic isolates have been classified as B. ranarum, B. meristosporus, and B. haptosporus. However, taxonomic studies based on antigenic analysis, isoenzyme banding, and restriction enzyme analysis indicated that all human pathogens belong to B. ranarum [3].

For ocular infections, only a few reports of this unusual pathogen exist in the literature. This present study describes a case of infectious scleritis caused by B. ranarum in a 47-yearold man from Northern Thailand.

\section{Case}

A 47-year-old male patient presented with a red eye, scleral nodule, and increased intraocular pressure (IOP) in the left eye for 3 weeks after an eye injury from sawdust. At a regional hospital, he was treated as nodular scleritis and secondary ocular hypertension for 1 week with topical prednisolone acetate $1 \%$ every $2 \mathrm{~h}$, nepafenac four times daily, timolol maleate $0.5 \%$ and brimonidine tartrate $0.1 \%$ twice daily, oral prednisolone $15 \mathrm{mg} /$ day, and oral acetazolamide $250 \mathrm{mg}$ twice daily. When the lesion did not improve, the patient was referred to a tertiary hospital. His best corrected visual acuity (BCVA) was 6/6 and 6/12 in OD and OS, respectively. Slit-lamp examination revealed a painless, slow-growing nodular lesion at the inferonasal area with marked injection of conjunctiva and mucopurulent discharge (Fig. 1a). There was a small infiltration of the adjacent peripheral cornea; otherwise, the anterior chamber, the lens, and fundus were unremarkable with an IOP of $20 \mathrm{~mm} \mathrm{Hg}$. The patient was diagnosed as infectious scleritis with scleral abscess and was admitted to hospital. Both topical and oral steroids were discontinued and a surgical drainage was performed. Yellow pus from the abscess was sent for microbial study including a potassium hydroxide wet mount, Calcofluor white staining, Gram staining, and bacterial and fungal cultures. The smears showed no organism and the culture did not grow any organism. The patient was initially treated as infectious scleritis with fortified cefazolin $(33 \mathrm{mg} / \mathrm{mL})$ and fortified gentamycin $(14 \mathrm{mg} / \mathrm{mL})$ every $2 \mathrm{~h}$ and oral ciprofloxacin $500 \mathrm{mg}$ twice daily along with previous anti-glaucoma medications. Eight days after treatment, the sclera nodule resolved, and the cornea was clear with controlled IOP, so the patient was discharged and instructed to follow up at the previous regional hospital. Two weeks later, his vision got worse, so he went to the regional hospital. At that time, increased corneal haze and anterior chamber reaction with plasmoid aqueous and vitreous haze in the left eye were noticed. The patient received fortified cefazolin and fortified gentamycin hourly to the left eye as well as systemic therapy with intravenous vancomycin $500 \mathrm{mg} /$ day and ceftazidime $1 \mathrm{~g} /$ day, before referral to a tertiary hospital.

At the second referral, BCVA were $6 / 6$ and hand motion in OD and OS, respectively. Slitlamp examination revealed markedly injected conjunctiva, diffused chemosis with mild proptosis, mucopurulent discharge, diffused corneal edema with neovascularization involving an area of previous infiltration, and marked anterior chamber reaction with plasmoid aqueous in the left eye (Fig. 1b). Since orbital cellulitis could not be ruled out, a computerized tomography scan of the orbit was performed, with the results showing localized preseptal swelling 


\section{Case Reports in Ophthalmology}

Case Rep Ophthalmol 2018;9:315-321

DOI: $10.1159 / 000489695$

(c) 2018 The Author(s). Published by S. Karger AG, Basel www.karger.com/cop

Tananuvat et al.: Ocular Basidiobolomycosis: A Case Report

and scleral thickening of the left eye (Fig. 1c). B-scan ultrasonography showed diffuse scleral thickening and exudative retinal detachment (Fig. 1d). The results of previous microbial workup demonstrated Basidiobolus species (Fig. 2). The patient was treated with ketoconazole $2 \%$ eye drops hourly, subconjunctival injection of fluconazole $1 \mathrm{mg}$ every other day, oral itraconazole $200 \mathrm{mg}$ daily, oral trimethoprim $400 \mathrm{mg}$, and sulfamethoxazole $80 \mathrm{mg} 2$ tablets twice daily. In addition, diabetes mellitus was identified during his general workup, thus antidiabetic drugs were given. Five days after treatment, his left eye developed more proptosis and limitation of extraocular movement, increased chemosis as well as diffused corneal stromal and subepithelial infiltration (Fig. 3). Corneal confocal microscopy was done and showed multiple round to oval-shaped lesions which looked like fungal zygospores in the corneal stroma (Fig. 3 inset). The patient then underwent exploratory surgical intervention. Multiple scleral abscesses were found. Pus from the abscess was sent for microbial study showing fungal hyphae and zygospores (Fig. 2a-c). The fungal culture also demonstrated Basidiobolus sp. and the nucleotide sequence indicated B. ranarum (Fig. 2d). Oral itraconazole was increased to $400 \mathrm{mg}$ daily. Eight days after treatment, the lesions seemed to respond to treatments as the proptosis, chemosis, and limitation of extraocular motions decreased, but generalized stromal and subepithelial infiltrations were still seen in the left eye. Nevertheless, the patient rejected further treatment and wanted to be treated as an outpatient. Two weeks after discharge, BCVA of his left eye was still hand motion despite an improvement of other signs including proptosis, chemosis, limitation of extraocular motions, and corneal infiltrations. Since then, the patient was lost to follow-up.

\section{Discussion}

Basidiobolus sp. is a saprophytic, filamentous fungus found in the soil, decaying vegetable matters as well as gastrointestinal tracts of amphibians, reptiles, fish, and bats [4]. It commonly causes infection of the skin [5], subcutaneous tissue [6-8], gastrointestinal tract $[9,10]$, lungs, sinus [1], palate, and turbinates in humans as well as in animals [2,11]. Basidiobolus infection is sporadically reported from all parts of the world, including Africa, Asia, the USA, and Latin America [2]; however, tropical and subtropical regions are endemic areas.

Only few ocular cases including one case of giant orbitofacial basidiobolomycosis have been reported from the northern part of Nigeria [12], one case of keratitis with endophthalmitis in a fungal keratitis series from Saudi Arabia [13], and one case of postoperative fungal endophthalmitis from India [14]. All cases of ocular infection were caused by B. ranarum. Our case presented with infectious scleritis with scleral abscess caused by B. ranarum, which initially resembled bacterial scleritis that partially improved with antibacterial therapy. Then the infection extended to become sclerokeratitis and orbital cellulitis. The risk factors of ocular infection in this patient may have been multiple including the exposure to this mold following minor trauma, previous treatment with corticosteroids, and undetected diabetes mellitus. In skin disease, the infection usually occurs after minor skin trauma or insect bites, and males are more frequently affected than females [15]. After contact with $B$. ranarum, this fungus can produce lipase and protease to hydrolyse fatty and protein constituents, thus aiding invasion into deeper tissue [2].

The clinical presentation of subcutaneous disease is quite characteristic and can be easily diagnosed as the lesion usually presents as a painless, slow growing, hardened firm nodule that expands and spreads locally. In contrast, ocular infection is difficult to diagnose, because its clinical presentation is nonspecific and indistinguishable from other causes of infectious 
scleritis or endophthalmitis. However, the atypical features of infectious scleritis in this case report was a painless, slow-growing scleral abscess, which was different from a common symptom of severe pain in most cases of scleritis. The definite diagnosis of basidiobolomycosis can be made by microbiological identifications from smear and isolated culture. Fungal elements of B. ranarum include hyphae and zygospores [2]. The hyphae are thin-walled, septate, haphazardly branched, and often surrounded by eosinophilic amorphous material in tissues (Splendore-Hoeppli phenomenon) of patients with subcutaneous skin infection. In addition to hyphae, zygospores may be present in the tissue as spheroidal bodies that measured up to $30 \mu \mathrm{m}$ in diameter. They have a thin outer wall, foamy cytoplasm, and a nucleus containing a large nucleolus with typically characteristic conjugation beaks.

Pharmaceutical agents that have been used to treat non-ocular infections with good response include systemic potassium iodide, trimethoprim sulfamethoxazole, and oral azoles, particularly itraconazole [15]. However, clinical experience of treating patients with ocular basidiobolomycosis is limited to only few cases. In our case report, the delay in diagnosis probably allowed the infection to spread into the cornea and orbital tissue resulting in sclerokeratitis and orbital cellulitis. Even though this patient seemed to respond to the multiple antifungal agents as well as the surgical drainage of an abscess, unfortunately, the patient rejected treatment and was lost to follow-up. Therefore, further evaluation was not possible.

\section{Conclusions}

B. ranarum is an uncommon ocular pathogen that leads to diagnostic confusion and poor treatment outcomes. Diagnosis requires awareness and consideration of its possibility in differential diagnosis of patients with infectious scleritis and scleral abscess.

\section{Statement of Ethics}

This study was approved by the Research and Ethics Committee, Faculty of Medicine, Chiang Mai University (study code: OPT-2560-04695) and written informed consent was obtained from the patient.

\section{Disclosure Statement}

None of the authors has any conflicts of interest concerning the case report.

\section{References}

1 Singh R, Xess I, Ramavat AS, Arora R. Basidiobolomycosis: a rare case report. Indian J Med Microbiol. 2008 Jul-Sep;26(3):265-7.

2 Gugnani HC. A review of zygomycosis due to Basidiobolus ranarum. Eur J Epidemiol. 1999 Nov;15(10):9239.

3 3. El-Shabrawi MH, Kamal NM, Jouini R, Al-Harbi A, Voigt K, Al-Malki T. Gastrointestinal basidiobolomycosis: an emerging fungal infection causing bowel perforation in a child. J Med Microbiol. 2011 Sep;60(Pt 9):1395402.

4 Okafor JI, Testrake D, Mushinsky HR, Yangco BG. A Basidiobolus sp. and its association with reptiles and amphibians in southern Florida. Sabouraudia. 1984;22(1):47-51. 
5 Atadokpédé F, Gnossikè J, Adégbidi H, Dégboé B, Sissinto-Savi de Tovè Y, Adéyé A, et al. Cutaneous basidiobolomycosis: seven cases in southern Benin. Ann Dermatol Venereol. 2017 Apr;144(4):250-4.

6 Kumar Verma R, Shivaprakash MR, Shanker A, Panda NK. Subcutaneous zygomycosis of the cervicotemporal region: due to Basidiobolus ranaram. Med Mycol Case Rep. 2012 Aug;1(1):59-62.

7 Anaparthy UR, Deepika G. A case of subcutaneous zygomycosis. Indian Dermatol Online J. 2014 Jan;5(1):514.

8 Kumaravel S, Bharath K, Rajesh NG, Singh R, Kar R. Delay and misdiagnosis of basidiobolomycosis in tropical South India: case series and review of the literature. Paediatr Int Child Health. 2016 Feb;36(1):52-7.

9 Vikram HR, Smilack JD, Leighton JA, Crowell MD, De Petris G. Emergence of gastrointestinal basidiobolomycosis in the United States, with a review of worldwide cases. Clin Infect Dis. 2012 Jun;54(12):1685-91.

10 Geramizadeh B, Heidari M, Shekarkhar G. Gastrointestinal basidiobolomycosis, a rare and under-diagnosed fungal infection in immunocompetent hosts: a review article. Iran J Med Sci. 2015 Mar;40(2):90-7.

11 Chiewchanvit S, Khamwan C, Pruksachatkunakorn C, Thamprasert K, Vanittanakom N, Mahanupab P, et al. Entomophthoromycosis in Maharaj Nakorn Chiang Mai Hospital. J Med Assoc Thai. 2002 Oct;85(10):108994.

12 Idemudia A, Adetokumbo A, Akinfenwa A. KE S. Giant orbitofacial basidiobolomycosis: diagnostic and management challenges in a resource limited environment. Sudan JMS. 2013;7(4):43-6.

13 Jastaneiah SS, Al-Rajhi AA, Abbott D. Ocular mycosis at a referral center in Saudi Arabia: A 20-year study. Saudi J Ophthalmol. 2011 Jul;25(3):231-8.

14 Annamalai R, Kindo A, Muthayya M. Postoperative fungal endophthalmitis due to Basidiobolus ranarum: report of a rare case. J Clin Ophthalmol Res. 2016;4(2):89-91.

15 Mathew R, Kumaravel S, Kuruvilla S, Varghese RG, Shashikala, Srinivasan S, et al. Successful treatment of extensive basidiobolomycosis with oral itraconazole in a child. Int J Dermatol. 2005 Jul;44(7):572-5. 


\section{Case Reports in Ophthalmology}
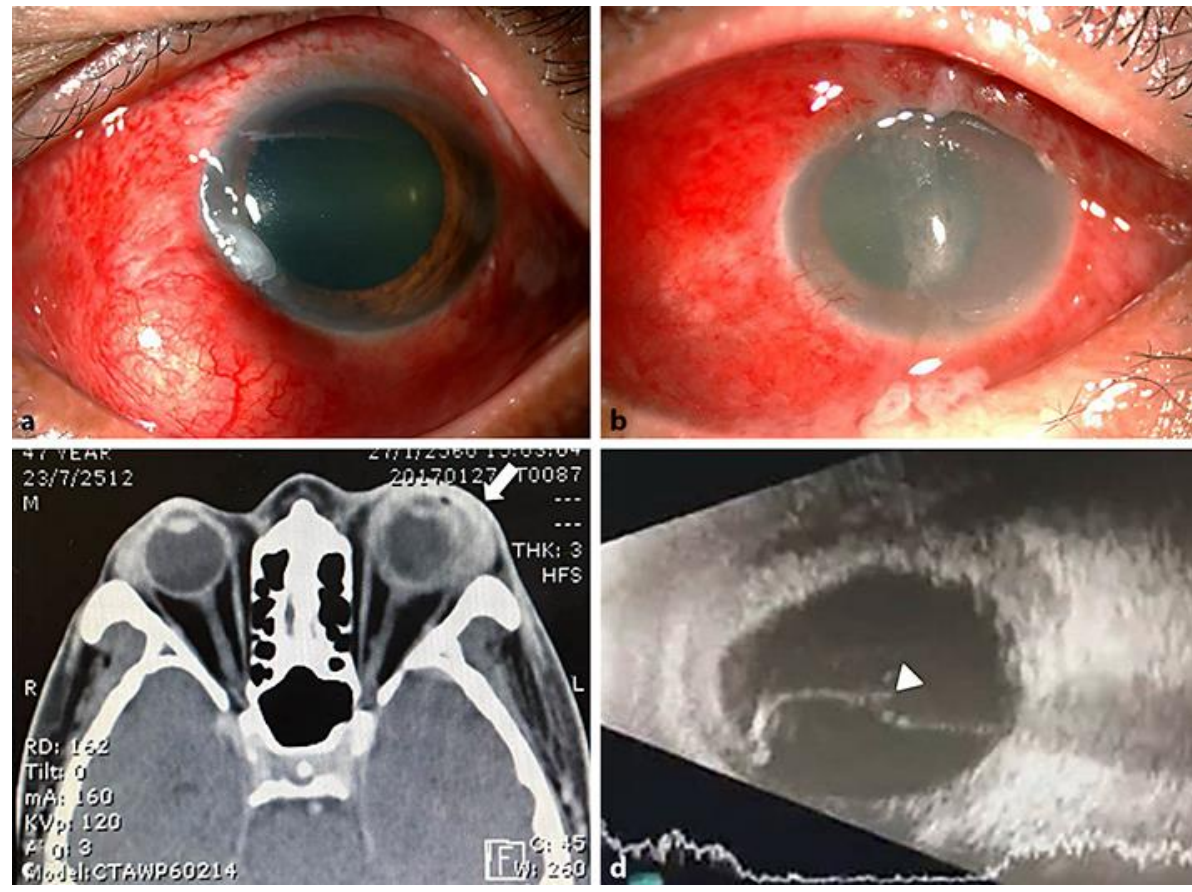

Fig. 1. Color photograph of the left eye. a Scleral nodule at the inferonasal region with marked diffused conjunctival injection and a small stromal infiltration of an adjacent peripheral cornea. $\mathbf{b}$ At second admission, the left eye had marked conjunctival injection, chemosis, mucopurulent discharge, diffused corneal edema with neovascularization involving an area of previous infiltration, and marked anterior chamber reaction. c Computerized tomography scan of the orbits shows diffused scleral thickening and localized soft tissue swelling at the anterolateral part of the globe in the left eye (arrow). $\mathbf{d}$ B-scan ultrasonography shows an exudative retinal detachment with fluid shifting in the left eye (arrowhead). 


\section{Case Reports in Ophthalmology}
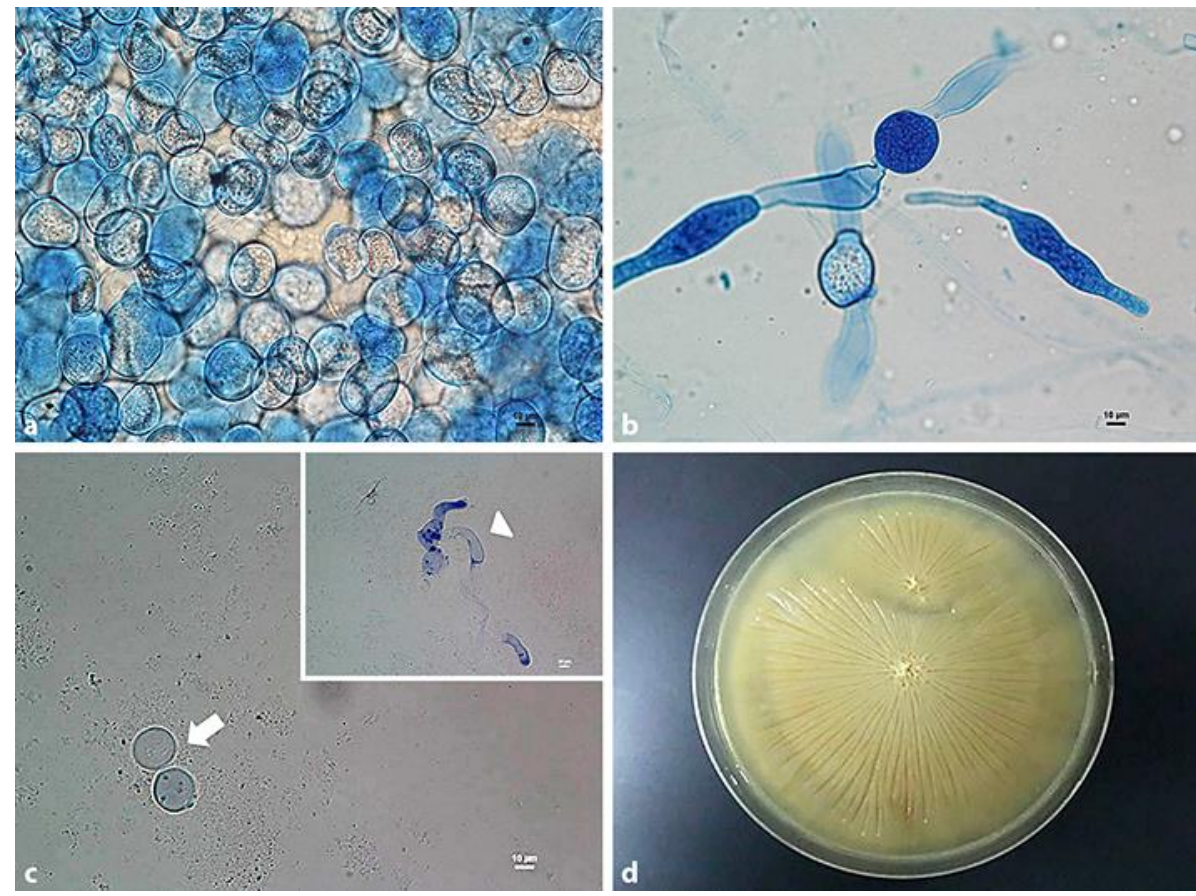

Fig. 2. Microscopic and gross features of B. ranarum. a Numerous zygospores are demonstrated in lactophenol cotton blue staining (400×). b Large sparsely septate hyphal elements are demonstrated in lactophenol cotton blue staining (400×). c Zygospores (arrow) and hyphae (arrowhead) are demonstrated in potassium hydroxide wet mount (400x). $\mathbf{d}$ Growth of yellowish colonies of B. ranarum with a radial folded appearance in Sabouraud dextrose agar.

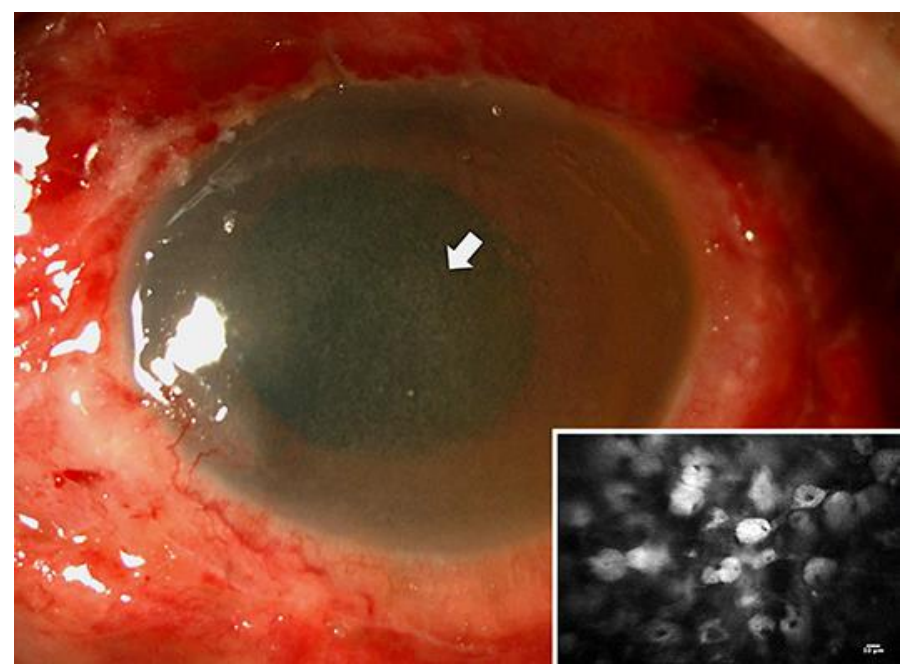

Fig. 3. Color photograph of the left eye shows diffused multiple dust-like subepithelial and stromal infiltrations in the left cornea. Inset Confocal microscopy findings demonstrate multiple round to oval-shaped lesions looking like zygospores of B. ranarum in the corneal stroma $(400 \times)$. 